This item was submitted to Loughborough's Research Repository by the author.

Items in Figshare are protected by copyright, with all rights reserved, unless otherwise indicated.

\title{
Forecasting oil and stock returns with a Qual VAR using over 150 years of data
}

PLEASE CITE THE PUBLISHED VERSION

http://dx.doi.org/10.1016/j.eneco.2017.01.001

PUBLISHER

(C) Elsevier

VERSION

AM (Accepted Manuscript)

\section{PUBLISHER STATEMENT}

This work is made available according to the conditions of the Creative Commons Attribution-NonCommercialNoDerivatives 4.0 International (CC BY-NC-ND 4.0) licence. Full details of this licence are available at: https://creativecommons.org/licenses/by-nc-nd/4.0/

\section{LICENCE}

CC BY-NC-ND 4.0

\section{REPOSITORY RECORD}

Gupta, Rangan, and Mark E. Wohar. 2019. "Forecasting Oil and Stock Returns with a Qual VAR Using over 150 Years of Data". figshare. https://hdl.handle.net/2134/26057. 


\title{
Forecasting Oil and Stock Returns with a Qual VAR using over 150 Years off Data*
}

\begin{abstract}
The extant literature suggests that oil price, stock price and economic activity are all endogenous and the linkages between these variables are nonlinear. Against this backdrop, the objective of this paper is to use a Qualitative Vector Autoregressive (Qual VAR) to forecast (West Texas Intermediate) oil and (S\&P500) stock returns over a monthly period of 1884:09 to 2015:08, using an in-sample period of 1859:10-1884:08. Given that there is no data on economic activity at monthly frequency dating as far back as 1859:09, we measure the same using the NBER recession dummies, which in turn, can be easily accommodated in a Qual VAR as an endogenous variable. In addition, the Qual VAR is inherently a nonlinear model as it allows the oil and stock returns to behave as nonlinear functions of their own past values around business cycle turning points. Our results show that, for both oil and stock returns, the Qual VAR model outperforms the random walk model (in a statistically significant way) at all the forecasting horizons considered, i.e., one- to twelve-months-ahead. In addition, the Qual VAR model, also outperforms the AR and VAR models (in a statistically significant manner) at long-run horizons for oil returns, and short- to medium-run horizons for stock returns.
\end{abstract}

JEL Classifications: C32, C53, C55, E32, G10, G17, Q41

Keywords: Vector Autoregressions, Business Cycle Turning Points, Forecasting, Oil and Stock Prices

\footnotetext{
${ }^{*}$ We would like to thank two anonymous referees for many helpful comments. However, any remaining errors are solely ours.
} 


\section{Introduction}

The aim of this paper is to forecast oil and stock returns using historical data spanning more than 150 years, by allowing for a role of movements in the real economy to affect and be affected by these markets in a nonlinear fashion. Put alternatively, our econometric framework allows us to simultaneously model endogeneity and nonlinearity in the relationship involving oil returns, stock returns and economic activity. In the process of forecasting oil and stock returns, we bring in the role of three relationships: (a) The oil market and economic activity; (b) The stock market and the real economy, and; (c) The oil and stock markets. Studies tend to concur that the oil market, the stock market and economic activity affect each other, i.e., they form an endogenous system, and this system operates in a nonlinear fashion. (Discussed in more detail in Section 2).

Against this backdrop, the objective of our paper is to forecast nominal Standard and Poor's 500 (S\&P 500) stock returns and nominal West Texas Intermediate (WTI) crude oil returns over the monthly period of 1884:08-2015:08, based on an in-sample period of 1859:10-1884:07, using a Qualitative Vector Autoregressive (Qual VAR) model. This longsample used by us essentially runs from the beginning of the modern era of the petroleum industry with the drilling of the first oil well on August 27, 1859 in Titusville, Pennsylvania; and is an unique feature of the paper. The forecasting performance of the Qual VAR model is compared with a random walk (RW) model; separate autoregressive (AR) model for oil and stock returns, and a standard Vector Autoregressive (VAR) model comprised of oil and stock returns. The in- and out-of-sample splits are statistically determined based on multiple structural break tests of Bai and Perron (2003), to ensure that all the breaks are restricted to the out-of-sample period, over which the models are recursively estimated. The recursive estimation allows us to accommodate for parameter changes in the models due to structural 
breaks. This provides a fair comparison with the nonlinear Qual VAR model, which due to the inherent nonlinearity in its design (as is discussed below in detail), would have captured the regime changes irrespective of how we decided to split the in- and out-of-samples. With the recursive estimation, even the linear models can account for structural changes as the parameter estimates get updated at each recursion, and hence accommodates for shifts in them due to breaks. Any gain from the Qual VAR will now be due to its ability to capture the nonlinearity in the relationship between the oil returns, stock returns and the measure of economic activity.

The VAR model, proposed by Sims (1980), has been shown to forecast macroeconomic and financial variables exceptionally well when compared to various other econometric models (Dueker, 2005; Dueker and Assenmacher-Wesche, 2010; Banbura et al., 2010; Giannone et al., 2015). The VAR is essentially a linear system of equations, whereby in a specific equation, a specific variable is regressed on the past values of itself and past values other variable(s) in this system, with possible allowance for deterministic terms (e.g., constant and trend). In other words, all variables comprising a VAR are endogenous. However, one criticism of the VAR forecasting is that variables in the model tend not to behave as linear functions of their own past values around business cycle turning points.

However, there is ample evidence that the relationship between oil returns, stock returns and economic activity is nonlinear (see Literature review for details). Given this, a linear VAR model is, at least theoretically, not suitable for our cause. In this regard, the Qual VAR model developed by Dueker (2005) helps us in two ways: First, the Qual VAR is essentially a regime-switching (hence, nonlinear) VAR model, based on the idea that the regime is determined by an observed qualitative response variable that is modelled simultaneously within the VAR. Models of this form consider a qualitative variable that is binary, and hence has two regimes such as the state of the business cycle. The advantage of this approach is that 
it allows one to understand which economic forces drive the regime switches. Second, given that there is no available data on monthly economic activity (like industrial production), dating as far back as 1859:10, the Qual VAR model allows us to use the monthly recession dummies (converted to an underlying continuous variable) to capture economic activity of the US economy. ${ }^{1}$ In the process, our Qual VAR model, over and above the stock and oil returns, includes a truncated normal latent business cycle index that is negative during National Bureau of Economic Research (NBER) recessions and positive during expansions. It must be pointed out that the nonlinearity between oil returns, stock returns, and a measure of economic activity (if available) could have been modelled using other nonlinear approaches ${ }^{2}$ such as the Markov-Switching VAR (originally developed by Hamilton (1989) in a univariate set-up), smooth threshold VAR (Leamer and Potter, 2002), and time-varying VAR (Primiceri, 2005). However, the fact that there is no data on economic activity over the sample period under consideration tilts the balance in favor of the Qual VAR model.

Following the suggestions of Banbura et al., (2010), we accommodate the problem of overparametrization (often leading to poor forecasts) in the VAR and the Qual VAR compared to the standard AR by using Bayesian shrinkage to ensure that all these models have the same in-sample fit and hence, a fair out-of-sample comparison. To the best of our knowledge, this is the first attempt to develop and forecast oil and stock returns with a Qual VAR, using data that spans over 150 years of history of these two important markets. In the process, we add to the literature on forecasting oil and stock prices or returns (see Huntington et al., (2013), Rapach and Zhou (2013) and Narayan and Gupta (2015) for detailed discussions of the literature on forecasting oil and stock prices (returns) respectively).

\footnotetext{
${ }^{1}$ US industrial production data at monthly frequency only starts from January of 1919. Though real GDP data is available from 1800, it is only available at annual frequency. In addition, a quarterly real GNP series starting in 1875:1 could be constructed by merging data from the NBER prior to 1947, and then from the FRED database of the Federal Reserve Bank of St. Louis.

${ }^{2}$ For a detailed discussion in this regard, the reader is referred to Balcilar et al., (2015).
} 
The remainder of the paper is organized as follows: Section 2 presents a brief literature review associated with the relationship between the oil market, the stock market and movements in economic activity. Section 3 discusses the methodology adopted in this paper, while Section 4 describes the data and presents the results from the forecasting exercise. Finally, section 5 concludes.

\section{A Brief Review of the Related Literature}

Following the seminal work of Hamilton (1983), a large literature exists that connects movements (in-sample and out-of-sample) in oil prices with recessions in the US economy. Hamilton (2011), and Baumeister and Kilian (2015), and Balcilar, Gupta, and Wohar (forthcoming) provides a detailed review. According to Hamilton (2008), nine of ten recessions in the US since World War II have been preceded by an increase in oil price. In fact, Hamilton (2009) even goes so far as to argue that a large proportion of the recent downturn in the US during the "Great Recession" can also be attributed to the oil price shock of 2007-2008. In the same vein, there is also a large literature that links movement in stock prices (again within and out-of-sample) with economic activity, dating as far back as Mitchell and Bums (1938). More recent studies followed, like Estrella and Mishkin (1998), Stock and Watson (2003), Rapach and Weber (2004) Nyberg (2011), and have been surveyed in detail in Erdogan et al., (2015), and Balcilar, Gupta and Wohar (forthcoming).

While, the general belief is that oil and stock prices are leading indicators of the economy, there is ample evidence that economic activity too plays a crucial role in predicting both in and out-of-sample movements in oil (see for example, Kilian (2009), Kilian and Vigfusson (2013), Baumeister and Kilian (2014, 2015) and Baumeister et al., (2015)) and stock prices (see for example, Rapach, Wohar and Rangvid (2005), Goyal and Welch (2008), Rapach, Strauss and Zhou (2010), Rapach and Zhou (2013)). More importantly, as 
highlighted, amongst others, by Baumeister et al., (2010), Kilian and Vigfusson (2011), Baumeister and Peersman (2013), Balcilar, Gupta and Miller (2015), Bjørnland and Larsen (2015) for the oil market, and Simo-Kengne et al., (2015), Tiwari et al., (2016) and Antonakakis et al., (forthcoming) for the stock market; these relationships with economic activity are in fact nonlinear.

At the same time, there also exists a large literature, as discussed in Kilian and Park (2009), Apergis and Miller (2009), and Balcilar, Gupta and Wohar (forthcoming), that relates (short and long-run) movements in oil and stock markets with economic activity through direct channels (cash-flow, investment, interest rate and exchange rate), and also indirectly. Again, this relationship between oil and stock markets is shown to be characterized more appropriately in a nonlinear fashion rather than a linear one (see for example, Antonakakis and Filis (2013), Balcilar and Ozdemir (2013), Antonakakis, et al., (2014), Boradstock and Filis (2014), Liu et al., (2015), Narayan and Gupta (2015), and Kang et al., (forthcoming)).

In sum, the oil market, the stock market and economic activity are in general endogenous to each other, with their relationship being more nonlinear rather than linear. Given this scenario, we aim to model the endogeneity and nonlinearity associated between these two important markets and economic activity while forecasting oil and stock returns over a historical sample period. Since a measure of economic activity like that of industrial production and GDP or GNP is not available over the 150 years of monthly data we are analysing here, we use the Qual VAR approach. The Qual VAR uses the qualitative recession dummies available over the period of our study to develop an underlying measure of economic activity allows the variables of interest to be related in an endogenous and nonlinear way. By comparing our forecasting results with linear models like the RW, AR and VAR models, we are able to judge the importance of the role played by the measure of economic activity related in an endogenous and nonlinear way with the oil and stock markets. 
Note that instead of relying on an in-sample evaluation of the Qual VAR relative to the other linear models, we pursue a forecasting exercise, since the ultimate test of any variable and/or predictive model is its out-of-sample performance (Campbell, 2008).

Related research involving forecasting of macroeconomic variables with Qual VARs can be found in Dueker (2005), Dueker and Assenmacher-Wesche (2010), and Gupta et al., (forthcoming). Using a Qual VAR model comprising of output, prices, interest rates, and the term spread (difference between the 10-year government bond yield and the 3-month Treasury bill rate), over and above a latent business cycle index, Dueker (2005) show that the US recession of March, 2001 could have been predicted with great success. Using the same framework, Dueker and Assenmacher-Wesche (2010) finds that the Qual VAR improves on out-of-sample forecasts from a standard VAR for, output, prices, interest rates and the termspread. Building on this line of work, more recently, Gupta et al., (forthcoming) develop a Factor-Augmented Qual VAR (FA-Qual VAR), where the model include information from a large data set in form of factors. The authors show that the FA-Qual VAR model outperforms the Qual VAR model of Dueker (2005), and Dueker and Assenmacher-Wesche (2010) at short to medium-run horizons when forecasting output, prices, interest rates and the term spread. Though not for forecasting, the Qual VAR approach has also been used to analyse causality between interest rates and state of the business cycle (Nyberg, 2013), and the impact of unconventional monetary policy (Meinusch and Tillmann, forthcoming; Tillmann; 2015, forthcoming).

\section{Forecasting Models and Metrics}

Since, the focus of the paper is to accommodate for endogeneity and nonlinearity in the relationship between oil returns, stock returns and economic activity, we start by describing the Qual VAR model used in forecasting oil and stock returns. Suppose we observe a qualitative variable, $y \in\{0,1\}$, which is driven by a continuous latent variable, $y^{*}$, such that: 
along with:

$$
\begin{aligned}
y_{t} & =0 \text { iff } y_{t}^{*} \leq 0 \\
& =1 \text { iff } y_{t}^{*}>0
\end{aligned}
$$

$$
y_{t}^{*}=\Psi(L) y_{t-1}^{*}+\Gamma(L) X_{t-1}+\epsilon_{t}
$$

with $\epsilon \sim N(0,1)$, and where $X_{t-1}$ is a set of explanatory variables (oil and stock returns in our case), and $\Psi(L)$ and $\Gamma(L)$ are lag polynomials. The qualitative data used for $y_{t}$ are the recession/expansion classifications designated by the business cycle dating committee at the NBER. Then, as described in Dueker (2005) and Dueker and Assenmacher-Wesche (2010), a Qual VAR model with $k$ variables and $p$ lags is expressed as a standard VAR:

$$
\Phi(L) Y_{t}=\epsilon_{t}
$$

with $\epsilon_{t} \sim \operatorname{Normal}(0, \Sigma)$, and where $Y_{t}=\left(\begin{array}{c}X_{t} \\ y_{t}^{*}\end{array}\right)$ is a $k \times 1$ vector consisting of oil and stock returns, i.e., $X_{t}$, plus the latent business cycle turning point index $y^{*} ; \Phi(L)$ is a set of $k \times k$ matrices from $L=0, \ldots, p$, with the identity matrix at $L=0$, i.e., the VAR regression coefficients are given in $\Phi(L)$. The parameters that require conditional distributions for Markov Chain Monte Carlo (MCMC) estimation are $\Phi, y^{*}$ and $\Sigma$ (i.e., the covariance matrix), which in turn, involves a sequence of draws from the following conditional distributions, where superscripts indicate the iteration number:

VAR coefficients $\sim$ Normal

$$
f\left(\Phi^{(i+1)} \mid\left\{y_{t}^{*(i)}\right\}_{t=1, . ., T},\left\{X_{t}\right\}_{t=1, . ., T}, \Sigma^{(i)}\right)
$$

Covariance matrix inverted Wishart

$$
f\left(\Sigma^{(i+1)} \mid\left\{y_{t}^{*(i)}\right\}_{t=1, . ., T},\left\{X_{t}\right\}_{t=1, . ., T}, \Phi^{(i)}\right)
$$

Latent variable $\sim$ truncated Normal 


$$
f\left(y_{t}^{*(i+1)} \mid \Phi^{(i+1)}, \Sigma^{(i+1)}\left\{y_{t}^{*(i+1)}\right\}_{j<t},\left\{y_{k}^{*(i)}\right\}_{k>t,}\left\{X_{t}\right\}_{t=1, \ldots, T}\right)
$$

Conditional on a set of values for $y^{*}, \Phi$ are normally distributed. While, $\Sigma$ is part of a normal-inverted Wishart conjugate pair with $\Phi$. Finally, each observation of $y^{*}$, has a truncated normal distribution, where it is not allowed to be negative (positive) during expansions (recessions). Further details on these conditional and prior distributions of the latent variable can be found in the Appendix of the paper, where the exposition follows closely of that of Dueker and Assenmacher-Wesche (2010).

Since our objective is to analyse the forecasting performance of the Qual VAR for oil and stock returns, we need alternative forecasting models as well. In this regard, we use three benchmarks, a random walk model (RW), a univariate autoregressive model of order $p(\operatorname{AR}(p))$, and a vector autoregressive model of order $p(\operatorname{VAR}(p))$. The $\operatorname{VAR}(\mathrm{p})$ model comprises of just the oil and stock returns, and looks exactly similar to equation (3), but now $Y_{t}=\left(X_{t}\right)$. In other words, each equation of the $\operatorname{VAR}(p)$ model comprises of $p$ lags of the oil and stock returns, besides a constant, as the predictors. The $\operatorname{AR}(p)$ model is the univariate version of the $\operatorname{VAR}(p)$, where the model for oil (stock) returns just comprises of p lags of oil (stock) returns as predictors, and a constant. Finally, the RW model, is just the constant mean model, i.e., the model contains only a constant (given that oil and stock returns are mean-reverting). Given the structures of the $\mathrm{RW}, \operatorname{AR}(p)$ and $\operatorname{VAR}(p)$ models, it is clear that the Qual VAR model nests all these three models.

To evaluate the forecast performances of these models, we use the Mean Square Forecast Error (MSFE), which in turn, is defined as follows:

$$
M S F E=(T-R-h+1)^{-1} \sum_{t=R}^{T-h}\left(u_{t+1}\right)^{2}
$$


where $T$ is the total sample, $R$ is number of observations used for estimation of the model from which the first forecast is formed (i.e. the in-sample portion of the total number of observations), $h$ is the forecasting horizon, and $u$ being the forecast error. It is important for us to check whether the Qual VAR model produces lower MSFEs than the RW, AR and VAR models; and also to check whether the forecasting performance is significantly better in the statistical sense. For this purpose, we use the powerful MSE-F statistic of McCracken (2007), since it is suited for nested models such as ours whereby the Qual VAR nests all its other competitors. The MSE-F statistic tests the null hypothesis that restricted (RW, AR and VAR) and unrestricted (Qual VAR) models have equal forecasting ability. The null is tested against the one-sided alternative hypothesis that the MSFE for the unrestricted model (Qual VAR) forecasts is less than the MSFE for the restricted model (RW, AR or VAR) forecasts. Formally, the statistic is given as:

$$
\begin{aligned}
& M S E-F=(T-R-h+1) \cdot \bar{d} / M S F E_{1} \\
& \bar{d}=M S F E_{0}-M S F E_{1}
\end{aligned}
$$

where $M S F E_{1}$ and $M S F E_{0}$ are the (MSFE) of the out-of-sample predictions from the unrestricted model and the specific restricted model (with which the comparison is made), respectively. A (positive) significant $M S E-F$ statistic indicates that the unrestricted model (Qual VAR) forecasts are statistically superior to those of a RW, AR or VAR, i.e., restricted model.

An additional point that needs to be discussed now is the issue of overparameterization in the VAR and the Qual VAR relative to the AR. To address this concern, we impose the standard Minnesota prior developed by Litterman (1986) on the parameters of the VAR and the Qual VAR. The Minnesota prior imposes restrictions on the coefficients of longer lags by assuming that these are more likely to be near zero than the coefficient on shorter lags. However, if there are strong effects from less important variables, the data can 
override this assumption. The restrictions are imposed by specifying normal prior distributions with zero means and small standard deviations for all coefficients with the standard deviation decreasing as the lags increases. The exception to this, however, is the coefficient on the first own lag of a variable, which has a mean of unity. But, if the variables in the VAR are stationary (which happens to be in our case), one needs to also impose a zero prior mean on the coefficient of the first own lag of a variable. ${ }^{3}$ The reader is referred to Litterman (1986) and Banbura et al., (2010) for the technical details. Since it is quite well-known in the forecasting literature now, it has been presented only briefly below:

$$
E\left[\left(\Phi_{k}\right)_{i j}\right]=\left\{\begin{array}{cc}
\delta_{i}, & j=i, k=1 \\
0, & \text { otherwise }
\end{array}, V\left[\left(A_{k}\right)_{i j}\right]=\left\{\begin{array}{cc}
\lambda^{2} / k^{2}, & j=i \\
\lambda^{2} \sigma_{i}^{2} / k^{2} \sigma_{j}^{2}, & \text { otherwise }
\end{array}\right.\right.
$$

In the Minnesota prior-settings, the hyperparameter $(\lambda)$ is used to determine how prior beliefs relate to the information contained in the data. More precisely, this hyperparameter controls the overall tightness of the prior distribution around the prior mean, $\delta_{i}$. Alternatively, $\lambda$ determines the importance of the prior beliefs in relation to the information contained in the data. When $\lambda=0$, the posterior equals the prior and the data exert no influence on the estimation. When $\lambda=\infty$, no influence of the prior exists and, hence, the parameter estimates coincide with the ordinary least squares (OLS) estimates. The factor $1 / k^{2}$ equals the rate by which the prior variance decreases as the lag length of the VAR increases, and $\sigma_{i}^{2} / \sigma_{j}^{2}$ accounts for the scale difference and data variability. The coefficient $\vartheta \in(0,1)$ governs the extent to which the lags of other variables are "less important” relative to the own lags. Following Banbura et al., (2010), we set $\vartheta=1$.

\footnotetext{
${ }^{3}$ A diffuse prior is used for the constants (i.e., $\Phi(0)$ ) in the equations of the VAR and Qual VAR models as in Banbura et al., (2010).
} 
Banbura et al., (2010), argue that the tightness-hyperparameter $(\lambda)$ should reflect the size of the system, i.e., as the number of variables increases, the parameters should shrink to avoid overfitting. This, in turn, is achieved by setting this hyperparameter to match the in-sample fit as the benchmark AR models of oil and stock returns. Understandably, the AR models have an infinite value for this hyperparameter with the corresponding values for the VAR and Qual VAR being 0.0821 and 0.0732 , and 0.1757 and 0.0732 for the cases of oil returns and stock returns respectively. These values of the hyperparameter in turn, ensures an in-sample fit of 0.2947 and 0.3887 - as obtained for the AR models of oil and stock returns respectively. ${ }^{4}$

\section{Data and Forecasting Results}

Our data set includes nominal values of the S\&P 500 index and WTI Crude oil price, covering the monthly period of 1859:09 to 2015:08. The raw data comes from the Global Financial database, which we seasonally adjust using the X-13 procedure of the US Census Bureau. Note that the MCMC approach of the Qual VAR requires that the data be stationary; hence, we work with nominal stock and oil returns (i.e., the first-differences of the natural logarithms of stock and oil prices expressed in percentages). The Data transformation implies that our effective sample starts from 1859:10-2015:08, with the end date and the starting point being driven purely by data availability at the time of writing this paper. Note that, the qualitative variable is a binary $0 / 1$ variable that denotes recessions and expansions, with switches taking place at business cycle turning points. The recession and expansion classification comes from the NBER. ${ }^{5}$

To determine the in-sample out-of-sample split, we first estimate a VAR model

\footnotetext{
${ }^{4}$ Based on the suggestion of an anonymous referee, we also produced forecasts from classical estimation of the VAR and the Qual VAR. However, these models produced consistently higher MSFEs relative to their Bayesian counterparts, thus highlighting the superiority of the Bayesian method. Complete details of these results are available upon request from the authors.

${ }^{5}$ The information of the dates of expansions and recessions areis available at: http://www.nber.org/cycles.html.
} 
comprising of oil and stock returns and determine the optimal lag-length based on the Akaike Information Criterion (AIC). The AIC suggested 5 lags. We then test for structural breaks in stock returns and the oil returns equations of the VAR(5) using the multiples structural break tests of Bai and Perron (2003). The oil returns equation picked up five breaks at: 1884:08, 1907:11, 1931:09, 1956:12, and 1986:04, while the stock returns equation showed only one break at 1933:06. Given that for the VAR(5) system as a whole, the earliest break was obtained at 1884:08, we chose an in-sample period of 1859:10 to 1884:07. Over the out-of-sample period of 1884:08-2015:08, the models are recursively estimated to produce forecasts at horizons $(h)$ of one-month-ahead to twelve-months-ahead At this stage, it is important to provide more details on the structure of our pseudo out-ofsample forecasting exercise for the Qual VAR. Keeping the lag-length of the VAR comprising of the stock and oil returns along with the recession/expansion dummy, fixed at 5, we extract the corresponding underlying latent business cycle indicator in a recursive way by adding one observation at a time over the out-of-sample period (1884:08-2015:05) for the variables of interest and the qualitative variable. This is an attempt to replicate a real-time situation that a forecaster faces, whereby the forecaster at the time of generating the first forecast, only has data available till 1884:07, i.e., the end-point of the in-sample. For this purpose, for each recursive estimation over the out-of-sample period, we use 10000 iterations from which the first 5000 are discarded to allow for convergence towards the posterior distribution. Figure 1 plots the latent business cycle indicator generated by the Qual VAR. As can be seen, the latent business cycle indicator picks up the various recessionary periods with great accuracy. Relatively deep recessions are picked up for the “Great Depression”, the oil price shock of 1973, and the recent “Great Recession”.

While creating the underlying qualitative variable in the Qual VAR, we produce forecasts for all the variables (oil and stock returns and the qualitative variable) in the model at each 
recursion over the out-of-sample period for horizons $(h)$ equal to one to twelve monthahead. We keep on doing this till the end of the sample period, giving us a total of 1573 one-step-ahead forecasts, 1572 two-step-ahead forecasts and so on, till we have 1562 twelve steps-ahead forecasts. The same iterative approach is also followed for the RW, AR(5), and standard VAR(5) models over the out-of-sample period to produce the forecasts at $h=1,2,3, \ldots . ., 12$. Note that we can only conduct a psuedo real-time forecasting analysis when a business cycle indicator is involved, since there is considerable time lag with which the NBER releases information that a turning point has occurred. Our data-vintage corresponds to 2015:09.

Table 1 presents the forecasting results for oil and stock returns at $h=1,2,3, \ldots . ., 12$ emanating from the AR, VAR, and the Qual VAR. The entries corresponding to the Qual VAR for each variable are MSFE of the Qual VAR relative to the Random Walk (RW) model. Hence a value less than one is indicative of the superior forecasting performance of the Qual VAR in comparison to the RW model, which in turn, is found to be the case for both oil and stock returns at all horizons. Entries corresponding to AR and VAR for both variables are MSFE of the AR and VAR relative to the Qual VAR model. ${ }^{6}$ Thus, in cases where these values are greater than one, it indicates that the Qual VAR outperforms the AR and VAR. As can be seen from Table 1 , for oil returns, this is the case at $h=8$ to 12 when compared to the AR and VAR. For the case of stock returns, the scenario is reversed in terms of horizons, with the Qual VAR outperforming the AR and VAR models for short- to medium-run horizons, i.e., for $h=1$ to 8 . For the horizons where the Qual VAR is not the best performing model, the evidence is slightly mixed in terms of the superior forecasting model. More specifically, for oil returns the AR model outperforms the VAR at $h=1$ and 7 , but for $h=2$ to

\footnotetext{
${ }^{6}$ It is easy to deduce from these ratios that the AR and VAR also outperform the RW model for $h=1 \ldots 12$, just like the Qual VAR does.
} 
6, the VAR is the best model. For stock returns, the performance of the AR and VAR is similar as is the best model for $h=10,11$ and 12, while the AR outperforms the AR at $h=9$.

Note that, as discussed earlier, the Qual VAR nests all the other models, i.e., the RW, AR and VAR. Given this, we use McCracken’s (2007) powerful MSE-F test statistic to determine whether the scenarios in which the Qual VAR outperforms the other models are significant or not. As indicated by the bold entries in the table, the MSE-F statistic is significant at the 5 percent level for all the cases where it outperforms the RW, AR and VAR for both oil and stock returns. So considering the performance of the Qual VAR, our results highlight the statistical importance of modelling nonlinearity through the use of the latent business cycle indicator at short- to medium-run horizons for stock returns, and at longer-run forecast steps for the oil returns.

\section{[Insert Table 1 Here]}

\section{Conclusions}

There exists a vast literature on the interrelationships between oil price, stock price and economic activity in the US (as well as the world). Reading of the literature tends to suggest that there are causal relationships running both ways amongst these three variables (i.e., these variables are all endogenous to each other), and these relationships are nonlinear in nature. Against this backdrop, the objective of this paper is to use a Qualitative Vector Autoregressive (Qual VAR) to forecast West Texas Intermediate oil and S\&P500 stock returns over a monthly period of 1884:09 to 2015:08, using an in-sample period of 1859:101884:08, with this split being determined by tests of multiple structural breaks. The unique feature of our data set is that it covers the entire modern era of the oil industry, given that first 
oil in the US was drilled in 1859:08 in Titusville, Pennsylvania. The Qual VAR helps us in two ways: (i) Given that there is no data on economic activity at monthly frequency dating as far back as 1859:09, we measure the same using the NBER recession dummies, which in turn, can be easily accommodated in a Qual VAR as an endogenous variable, unlike in a standard VAR; and, (ii) The Qual VAR is inherently a nonlinear model as it allows the explanatory variables in it to behave as nonlinear functions of their own past values around business cycle turning points - again something not possible in the standard VAR model, but is important for our purpose, given that the literature suggests that, oil price, stock price and economic activity are related in a nonlinear fashion.

Our results show that the Qual VAR model outperforms the random walk (RW) model for one- to twelve-months-ahead forecasts for both oil and stock returns. In addition, when we compare the Qual VAR model with AR and VAR models, we observe the following: (i) For oil returns, the Qual VAR outperforms the AR and VAR models at longer horizons, i.e., forecast horizons $(h)=8$ to 12 when compared to the AR and VAR consistently in a statistically significant manner; and, (ii) For the case of stock returns, the Qual VAR outperforms the AR and VAR models in a statistically significant way, for short- to mediumrun horizons, i.e., for $h=1$ to 8. So considering the performance of the Qual VAR, our results highlight the importance of modelling nonlinearity through the use of the latent business cycle indicator when forecasting oil and stock returns, especially at longer-run horizons for the former and short- to medium-run horizons for the latter. As part of future research, given that the VAR and Qual VAR are estimated using Bayesian methods, we can analyse the whole distribution of the forecasts for oil and stock returns. 


\section{References}

Antonakakis, N. and Filis, G. (2013). Oil prices and stock market correlation: a time-varying approach. International Journal of Energy and Statistics, 1 (1), 17-29.

Antonakakis, N., Chatziantoniou, I., Filis, G. (2014). Spillovers between oil and stock markets at times of geopolitical unrest and economic turbulence, MPRA Working Paper No. 59760.

Antonakakis, N., Andre, C., and Gupta, R. (Forthcoming). Dynamic Spillovers in the United States: Stock Market, Housing, Uncertainty and the Macroeconomy. Southern Economic Journal.

Apergis, N., Miller, S. M. (2009). Do structural oil-market shocks affect stock prices? Energy Economics, 31(4), 569-575.

Bai, J. and Perron, P. (2003). Computation and analysis of multiple structural change models. Journal of Applied Econometrics, 6, 72-78.

Balcilar, M., Ozdemir, Z. A. (2013). The causal nexus between oil prices and equity market in the US: A regime switching model, Energy Economics, 39, 271-282.

Balcilar, M., Gupta, R., Miller, S. M. (2015). Regime switching model of US crude oil and stock market prices: 1859 to 2013, Energy Economics, 49, 317-327.

Balcilar, M., Gupta, R., Majumdar, A., Miller, S.M. (2015). Was the recent downturn in US real GDP predictable? Applied Economics, 47(28), 2985-3007.

Balcilar, M., Gupta, R., Wohar, M. E. (Forthcoming). Common cycles and common trends in the stock and oil markets: Evidence from more than 150 years of data. Energy Economics.

Banbura, M., Giannone, D., and Reichlin, L. (2010). Large Bayesian vector auto regressions. Journal of Applied Econometrics 25(1), 71-92.

Baumeister, C., Peersman, G., and van Robays, I. (2010). The Economic Consequences of Oil Shocks: Differences across Countries and Time. In Fry, R., Jones, C., and Kent, C. (eds.), Inflation in an Era of Relative Price Shocks, Sydney, 91-128.

Baumeister, C., and Peersman, G. (2013). Time-varying effects of oil supply shocks on the US economy, American Economic Journal: Macroeconomics, 5(4), 1-28.

Baumeister, C., and Kilian, L. (2014). What Central Bankers Need to Know about Forecasting Oil Prices, International Economic Review, 55(3), 869-889.

Baumeister, C., and Kilian, L. (2015a). Forecasting the Real Price of Oil in a Changing World: A Forecast Combination Approach, Journal of Business and Economic Statistics, 33(3), 338-351.

Baumeister, C., Kilian, L. (2015b). 40 years of oil price fluctuations: why the price of oil may still surprise us, Mimeo, University of Notre Dame.

Baumeister, C., Kilian, L., and Lee, T.K. (2015). Are There Gains from Pooling Real-Time Oil Price Forecasts? Energy Economics, 46, S33-S43.

Bjørnland, H., Larsen, V. H. (2015). Oil and macroeconomic (in) stability. CAMP, Working Paper No. 201507.

Broadstock, D.C. and Filis, G. (2014). Oil price shocks and stock market returns: New evidence from the United States and China. Journal of International Financial Markets, Institutions and Money, 33, 417-433.

Campbell, J.Y., (2008) Viewpoint: estimating the equity premium, Canadian Journal of Economics, 41, 1-21.

Dueker, M. J. (2005). Dynamic forecasts of qualitative variables: a Qual VAR model of US recessions, Journal of Business and Economic Statistics, 23, 96-104.

Dueker, M. and Assenmacher-Wesche, K. (2010). Forecasting macro variables with a Qual VAR business cycle turning point index, Applied Economics, 42, 2909-2920. 
Erdogan, O., Bennett, P., Ozyildirim, C. (2015). Recession Prediction Using Yield Curve and Stock Market Liquidity Deviation Measures, Review of Finance, 19(1), 407-422.

Estrella, A., Mishkin, F. S., (1998). Predicting U.S. Recessions: Financial Variables as leading Indicators, Review of Economics and Statistics 80, 45-61.Giannone, D., Lenza, M., and Primiceri, G.E. (2015). Prior Selection for Vector Autoregressions. The Review of Economics and Statistics, 97(2), 436-451.

Goyal, A., and Welch, I. (2008). A Comprehensive Look at the Empirical Performance of Equity Premium Prediction. Review of Financial Studies, 21(4), 1455-1508.

Gupta, R., Olson, E., and Wohar, M.E., (Forthcoming). Forecasting Key US Macroeconomic Variables with a Factor-Augmented Qual VAR. Journal of Forecasting.

Hamilton, J. D. (1983). Oil and the macroeconomy since World War II, Journal of Political Economy, 91, 228-248.

Hamilton, J. D. (1989). A new approach to the economic analysis of nonstationary time series and the business cycle, Econometrica, 57, 357-84.

Hamilton, J. D. (2008). Oil and the macroeconomy. In New Palgrave Dictionary of Economics, 2nd edition, edited by Steven Durlauf and Lawrence Blume, Palgrave McMillan Ltd.

Hamilton, J. D. (2009). Causes and consequences of the oil shock of 2007-08, Brookings Papers on Economic Activity, Spring, 215-259.

Hamilton, J. D. (2011). Historical oil shocks. National Bureau of Economic Research, Working Paper No. w16790.

Huntington, H., Al-Fattah, S.M., Zhou, H., Gucwa, M., and Nouri, A. (2013). Oil markets and price movements: A survey of models, USAEE Working Paper, 13-129.

Kang, W., Ratti, R.A., Yoon, K.H., (Forthcoming). Time-Varying Effect of Oil Market Shocks on the Stock Market, Journal of Banking and Finance.

Kilian, L. (2009). Not All Oil Price Shocks Are Alike: Disentangling Demand and Supply Shocks in the Crude Oil Market, American Economic Review, 99(3), 1053-1069.

Kilian, L., Park, C. (2009). The impact of oil price shocks on the us stock market, International Economic Review, 50(4), 1267-1287.

Kilian, L., Vigfusson, R. J. (2011). Nonlinearities in the oil price-output relationship, Macroeconomic Dynamics, 15(S3), 337-363.

Kilian, L., Vigfusson, R. J. (2013). Do oil prices help forecast us real GDP? The role of nonlinearities and asymmetries, Journal of Business \& Economic Statistics, 31(1), 7893.

Leamer, E. and Potter, S. (2004) A nonlinear model of the business cycle. Paper presented at the Econometric Society 2004 North American Winter Meeting.

Litterman, R.B., (1986). Forecasting with Bayesian Vector Autoregressions - Five Years of Experience. Journal of Business and Economic Statistics 4(1), 25-38.

Liu, L., Ma, F., and Wang, Y. (2015). Forecasting excess stock returns with crude oil market data, Energy Economics 48, 316-324.

McCracken, M. W., 2007. Asymptotics for out-of-sample tests of Granger causality. Journal of Econometrics 140, 719-752.

Meinusch, A., and Tillmann, P. (Forthcoming). The macroeconomic impact of unconventional monetary policy shocks. Journal of Macroeconomics.

Narayan, P. K., Gupta, R. (2015). Has oil price predicted stock returns for over a century?. Energy Economics, 48, 18-23.

Nyberg, H. (2011). Forecasting the direction of the US stock market with dynamic binary probit models, International Journal of Forecasting, 27(2), 561-578.

Nyberg, H. (2013). A Qualitative Response VAR Model: An Application to Joint Dynamics of U.S. Interest Rates and Business Cycle. HECER Discussion Papers No. 369. 
Primiceri, G. E. (2005) Time Varying Structural Vector Autoregressions and Monetary Policy. Review of Economic Studies, 72, 821-852.

Rapach, D. E., Weber, C. E. (2004). Financial Variables and the Simulated Out-of-Sample Forecastability of US Output Growth since 1985: An Encompassing Approach, Economic Inquiry, 42(4), 717-738.

Rapach, D.E., Wohar, M.E., and Rangvid J. (2005). Macro Variables and International Stock Return Predictability. International Journal of Forecasting, 21(1), 137-166.

Rapach, D.E., Strauss, J.K., and Zhou, G. (2010). Out-of-Sample Equity Premium Prediction: Combination Forecasts and Links to the Real Economy, Review of Financial Studies, 23 (2), 821-862.

Rapach, D.E., and Zhou, G., (2013). Forecasting Stock Returns. In Handbook of Economic Forecasting, Volume 2A, Graham Elliott and Allan Timmermann (Eds.) Amsterdam: Elsevier, 328-383.

Simo-Kengne, B.D., Miller, S.M., Gupta, R., and Aye, G.C. (2015). Time-Varying Effects of Housing and Stock Prices on U.S. Consumption. Journal of Real Estate Finance and Economics, 50 (3), 339-354.

Sims, C. A. (1980). Macroeconomics and Reality. Econometrica, vol. 48, 1-48.

Stock, J. H., Watson, M. W. (2003). Forecasting Output and Inflation: The Role of Asset Prices, Journal of Economic Literature, 41, 788-829.

Tillman, P. (2015). Estimating the effects of macroprudential policy shocks: A Qual VAR approach, Economics Letters 15, 1-4.

Tillman, P. (Forthcoming). Unconventional monetary policy shocks and the response of emerging markets. Journal of International Money and Finance.

Tiwari, A.K., Albulescu, C.T., and Gupta, R. (2016). Time-frequency relationship between US output with commodity and asset prices. Applied Economics 48(3), pages 227242. 
Table 1. Forecasting results from AR, VAR and Qual VAR: 1884:08-2015:08

\begin{tabular}{|c|c|c|c|c|c|c|c|c|c|c|c|c|c|}
\hline & & \multicolumn{12}{|c|}{$h$} \\
\hline Variable & Model & 1 & 2 & 3 & 4 & 5 & 6 & 7 & 8 & 9 & 10 & 11 & 12 \\
\hline \multirow{3}{*}{ Oil Returns } & Qual VAR & 0.690 & 0.547 & 0.518 & 0.495 & 0.470 & 0.468 & 0.492 & 0.476 & 0.479 & 0.516 & 0.527 & 0.502 \\
\hline & AR & 0.984 & 0.996 & 0.998 & 0.996 & 0.997 & 0.999 & 0.999 & 1.001 & 1.004 & 1.006 & 1.006 & 1.005 \\
\hline & VAR & 0.996 & 0.992 & 0.993 & 0.993 & 0.994 & 0.997 & 0.999 & 1.001 & 1.003 & 1.006 & 1.006 & 1.005 \\
\hline \multirow{3}{*}{$\begin{array}{c}\text { Stock } \\
\text { Returns }\end{array}$} & Qual VAR & 0.547 & 0.492 & 0.465 & 0.511 & 0.543 & 0.489 & 0.509 & 0.524 & 0.525 & 0.509 & 0.503 & 0.509 \\
\hline & AR & 1.017 & 1.015 & 1.010 & 1.011 & 1.006 & 1.008 & 1.003 & 1.001 & 0.996 & 0.993 & 0.993 & 0.993 \\
\hline & VAR & 1.013 & 1.010 & 1.007 & 1.008 & 1.004 & 1.005 & 1.002 & 1.001 & 0.997 & 0.993 & 0.992 & 0.993 \\
\hline
\end{tabular}

Note: Entries corresponding to Qual VAR for each variable are MSFE of the Qual VAR relative to the Random Walk (RW) model; Entries corresponding to AR and VAR for each variable are MSFE of the AR and VAR relative to the Qual VAR model. Entries in bold indicates significance of McCracken's (2007) MSE-F statistic at the 5 percent level. 
Figure 1. Plot of the Latent Business Cycle indicator from the Qual VAR: 1859:10-2015:08

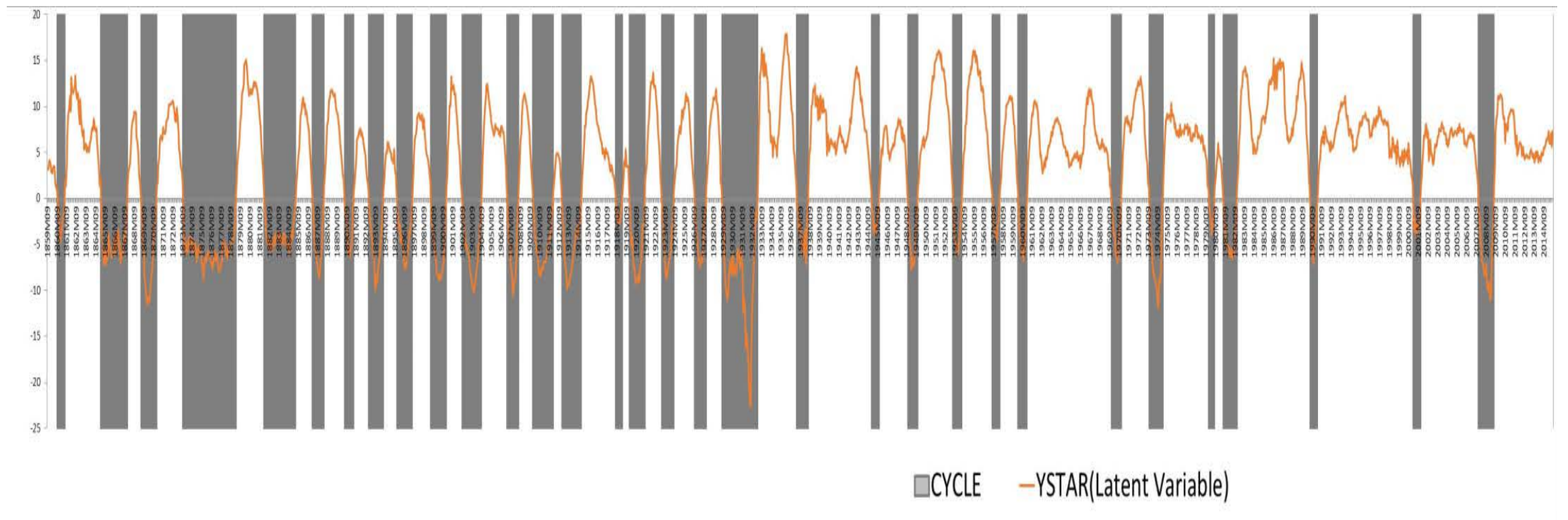

Note: CYCLE stands for NBER recession dates, and YSTAR stands for the latent continuous business cycle indicator ( $\left.y^{*}\right)$. 


\section{Appendix: Qual VAR Estimation}

Following Dueker and Assenmacher-Wesche (2010), here we present the mean and variance of the underlying normal distribution for the latent variable $\left(y^{*}\right)$. Based on $Y$ as the dataset which follows a $\operatorname{VAR}(p)$ process, we need to obtain the conditional distribution of $y_{i t} \mid Y_{-t}, X_{t}$, where $Y_{-t}$ is the full vector time series except for time $t$ data and $X_{t}$ is the vector at time $t$ except for the latent variable $y_{t}^{*}$.

We start off by obtaining the conditional distribution of $Y_{t} \mid Y_{-t}$. Since we have an autoregressive order of $p, Y_{t}$ will affect the residuals for the $p+1$ periods:

$$
\begin{gathered}
\epsilon_{t}=Y_{t}-\mu-\phi_{1} Y_{t-1}-\ldots-\phi_{p} Y_{t-p} \\
\epsilon_{t+1}=Y_{t+1}-\mu-\phi_{1} Y_{t}-\ldots-\phi_{p} Y_{t-p+1} \\
\vdots \quad \vdots \\
\epsilon_{t+p}=Y_{t+p}-\mu-\phi_{1} Y_{t+p-1}-\ldots-\phi_{p} Y_{t}
\end{gathered}
$$

Let $\kappa_{j}$ denote the known part of $\epsilon_{j}$ based on the assumption that the values of the latent variable from other periods are taken as given:

$$
\begin{gathered}
\epsilon_{t}=Y_{t}+\kappa_{t} \\
\epsilon_{t+1}=\kappa_{t+1}-\phi_{1} Y_{t} \\
\vdots \quad \vdots \\
\epsilon_{t+p}=\kappa_{t+p}-\phi_{p} Y_{t}
\end{gathered}
$$

The density of $\left(\epsilon_{t}, \ldots, \epsilon_{t+p}\right)$ can then be written as a function of $Y_{t}$ :

$$
\begin{aligned}
& -\frac{1}{2}\left(Y_{t}+\kappa_{t}\right)^{\prime} \Sigma^{-1}\left(Y_{t}+\kappa_{t}\right) \\
& -\frac{1}{2}\left(\kappa_{t+1}-\phi_{1} Y_{t}\right)^{\prime} \Sigma^{-1}\left(\kappa_{t+1}-\phi_{1} Y_{t}\right) \\
& \vdots \\
& -\frac{1}{2}\left(\kappa_{t+p}-\phi_{p} Y_{t}\right)^{\prime} \Sigma^{-1}\left(\kappa_{t+p}-\phi_{p} Y_{t}\right),
\end{aligned}
$$


where $\sum$ is defined as the cross-equation covariance matrix of the errors, which are uncorrelated across time.

After taking together all cross-products, we have

$$
Y_{t} \mid Y_{-t} \sim N\left(C^{-1} D, C^{-1}\right),
$$

where

$$
C=\left(\Sigma^{-1}+\phi_{1}^{\prime} \Sigma^{-1} \phi_{1}+\ldots+\phi_{p}^{\prime} \Sigma^{-1} \phi_{p}\right)
$$

and

$$
D=\left(-\Sigma^{-1} \kappa_{t}+\phi_{1}^{\prime} \Sigma^{-1} \kappa_{t+1}+\ldots+\phi_{p}^{\prime} \Sigma^{-1} \kappa_{t+p}\right) .
$$

For the sake of convenience, we define $\bar{Y}_{t}=Y_{t}-C^{-1} D$, where

$$
f\left(\bar{Y}_{t}\right) \propto \exp \left\{-.5 \bar{Y}_{t}^{\prime} C \bar{Y}_{t}\right\}
$$

We can assume that $y_{t}^{*}$ is the last element in $Y_{t}$, without any loss of generality, and partition $C$ accordingly:

$$
C=\left(\begin{array}{ll}
C_{00} & C_{01} \\
C_{01}^{\prime} & C_{11}
\end{array}\right)
$$

After collecting all the terms, we have

$$
\bar{y}_{t}^{*} \mid \bar{X}_{t} \sim N\left(-C_{11}^{-1} C_{01} \bar{X}_{t}, C_{11}^{-1}\right) .
$$

The conditional mean of $y_{t}^{*}$ is the conditional mean of $\bar{y}_{t}^{*}$ plus the bottom right-hand element of $C^{-1} D$. 